\title{
Research on Non-conservative Phenomena in Physics
}

\author{
Poshan He \\ Chongqing Vocational College of Transportation, Chongqing 401120, China. \\ Email:2502426407@qq.com
}

\begin{abstract}
Non-conservative phenomena are very common and very important in physics. They have the characteristic of work divergence (if an object moves under non-conservative force, the work it does is related to the path) and this phenomenon does not satisfy the time reversal invariance. Therefore, they can cause many situations. From a mathematical point of view, they are similar to divergence in infinite series. Therefore, the infinite series can analyze non-conservative phenomena. This paper takes non-conservative force as an example to prove the relationship between non-conservative force and infinite series.
\end{abstract}

Keywords: non-conservative phenomenon, infinite series, dissipation process

\section{Introduction}

As we know, many phenomena are dissipative in actual physical processes, and they will cause many situations that make them unable to obey time reversal invariance. Obviously, friction is a non-conservative force. If an object moves on other objects, the amount of mechanical work done is related to the route. Therefore, if friction is applied to an object, the number of times the object does work depends on the distance the object travels. This is the general conclusion that is not conservative. This article will firstly take friction as an example to introduce the common attributes of non-conservative phenomena, and then generalize to any non-conservative phenomena and prove the common attributes of non-conservative phenomena.

\section{The attributes of non-conservative force}

The phenomenon of friction is common and familiar. For example, when two objects touch and move with each other, the surfaces of the two objects will produce a kind of resistance, that is, friction. The strength of friction depends on the speed of relative movement. If the surface roughness of the object is not uniform, or if the object travels through a different route, then another object may experience different friction strength.

For two-dimensional to conservative forces, it obeys Gauss's law:

$$
\oiint_{\partial S} \frac{\partial^{2} \mathbf{F}}{\partial x \partial y} d l_{x} d l_{y}=\iiint_{V} \frac{\partial^{3} \mathbf{F}}{\partial x \partial y \partial z} d l_{x} d l_{y} d l_{z}
$$

Among them, "F" is force. It has three components in three dimensions, so it can work in every dimension.

For the conservative force, if it reverses in time, (1) is also true, because the conservative force obeys time reversal invariance:

$$
\frac{1}{-t} \oiint_{\partial S} \frac{\partial^{2} \mathbf{F}}{\partial x \partial y} d l_{x} d l_{y}=\frac{1}{-t} \iiint_{V} \frac{\partial^{3} \mathbf{F}}{\partial x \partial y \partial z} d l_{x} d l_{y} d l_{z}
$$

Therefore, for conservative forces, (1) and (2) are equivalent. For any conservative force, the relationship of functions can be found. Therefore, for a certain conservative force, if it is variable, it has only one behavior. Therefore, it is convergent, and if it works on a route, it must notice that it has nothing to do with the direction of the force:

$$
\lim _{n \rightarrow \infty} \mathbf{F}_{n} s=-\lim _{n \rightarrow \infty} \mathbf{F}_{n} s
$$

For non-conservative forces, (1) and (2) are not equivalent. This is due to the divergence of non-conservative forces, so it cannot find a potential function (analytic function), which makes it unable to obey Gauss's law: 
$\frac{1}{t} \oiint_{\partial S} \frac{\partial^{2} \mathbf{F}}{\partial x \partial y} d t_{x} d t_{y}=\frac{1}{t} \iiint_{V} \frac{\partial^{3} \mathbf{F}}{\partial x \partial y \partial z} d l_{x} d l_{y} d l_{z} \neq \frac{1}{-t} \iiint_{V} \frac{\partial^{3} \mathbf{F}}{\partial x \partial y \partial z} d l_{x} d l_{y} d l_{z}$

For the resultant force, it can be equivalent to the sum of the micro-elements of the force in three dimensions:

$$
E=\sum_{i=1}^{n} d w_{x i}+\sum_{i=1}^{n} d w_{y i}+\sum_{i=1}^{n} d w_{z i}
$$

Since it cannot find an analytic function, if it converges, it cannot converge to the same constant, so that it is uncertain in any different behavior, so it is divergent.

Analogous to the idea of path integral in quantum mechanics, the case of non-analytic functions is equivalent to the sum of infinitely many analytic functions, and it is also equivalent to the divergence of infinite series. Therefore, for the case of non-conservative forces, it is equivalent to the sum of an infinite number of conservative forces:

$$
\mathbf{F}^{D}=\sum_{i=1}^{\infty} \mathbf{f}^{C}
$$

For a particle system, assuming that the conservative force is gravity, the sum of the gravity of the particle system is divergent.

$$
\mathbf{F}^{D}=G \frac{\sum_{i=1}^{n} M_{i} m_{i}}{\sum_{i=1}^{n} r_{i}^{2}}
$$

And has an infinite potential function:

$$
-\nabla \cdot \mathbf{F}^{D}=G \frac{\sum_{i=1}^{n} M_{i}}{\sum_{i=1}^{n} \mathbf{r}_{i}} \rightarrow \infty
$$

The effect of the sum of infinitely many potential functions causes the divergence of the potential function and makes the potential function meaningless.

\section{Conservation of energy in non-conservative forces}

As mentioned earlier, the conservative force obeys time reversal invariance when doing work, and it obeys the law of conservation of energy, and because it has an analytic function, it can predict the state of energy at any time. Since work is conservative, this force is usually conservative. When a conservative force does work along any different path, energy is always conserved. This is the reason why the conservative force satisfies time reversal invariance. Due to the uncertainty of non-conservative force, the intensity of energy can only be determined at each position. But the energy of each position is conserved. Obey Gauss's law:

$$
\nabla \cdot \frac{\partial^{2} E_{i}}{\partial x \partial y}=\frac{\partial^{3} E_{i}}{\partial x \partial y \partial z} \text { ("i"is the position) }
$$

But the sum of energy has arbitrary behavior toward infinity in the particle system. When energy diverges, the moment of momentum also diverges. So according to (9):

$$
\sum_{i=1} \oiint_{\partial S} \frac{\partial^{2} \mathbf{P}_{i}}{\partial x \partial y} d x d y=\sum_{i=1} \iiint_{V} \frac{\partial^{3} \mathbf{P}_{i}}{\partial x \partial y \partial z} d x d y d z \rightarrow \infty
$$

The momentum is dispersed in every point, but it can continuously vary in every component. Therefore, the sum of momentum is divergent:

$$
\lim _{i \rightarrow \infty} m \sum_{i} v_{i}=\iiint_{i} P_{i} d t \rightarrow \infty
$$

The particle system has symmetry in the low-energy state, but loses symmetry in the high-energy state. Therefore, we 
can think of non-conservative force as a state in which energy is higher than conservative force. According to the second law of thermodynamics, it is impossible for any system to absorb heat from a single heat source to do work, so that it can be completely converted into useful work without any other effects. But this law is wrong in a high-energy environment, because the system has severe quantum effects and energy divergence in the high-energy state:

$$
\lim _{n \rightarrow 0} E_{n}=\infty
$$

\section{The nature of non-conservative power}

Since the non-conservative force cannot obey the energy conservation of the continuous process, it cannot converge to a constant. The essence of non-conservative force is that external force causes abnormal molecular behavior. ${ }^{[4]}$ At the micro level, the essence of non-conservative force is the random movement of particles. For example, friction is a nonconservative force, it can produce thermal effects, it stimulates the kinetic energy in the particles, causing them to appear irregular behavior. Any non-conservative phenomenon is accompanied by a dissipation process. This process is dissipative, and the result is the dissipation of energy in many particle systems.

\section{Examples of non-conservative forces}

As we all know, the Coulomb force is a conservative force, and it has an analytic function:

$$
F=k \frac{Q}{r^{2}}
$$

Its value converges to a constant, but it is not suitable for many free electronic spaces. In this space, the Coulomb force diverges:

$$
\lim \sum F=\frac{k}{r^{2}} \oiint_{i} Q_{i} \rightarrow \infty
$$

In this case, the intensity or energy in the electromagnetic field is divergent, which leads to a change in Maxwell's equation:

$$
\left\{\begin{array}{l}
\oint_{\partial s} \mathbf{D} \cdot d s=\int_{v} \rho d v+\int_{v} \frac{\partial \rho}{\partial t} d v \\
\oint_{\partial S} B \cdot d S=0 \\
\int_{l} \mathbf{E} \cdot d l=-\frac{d}{d t} \int_{s} \mathbf{B} \cdot d \mathbf{s}+\int_{s} \frac{d \mathbf{B}}{d t} \cdot d \mathbf{s} \\
\int_{l} \mathbf{H} \cdot d l=\int_{s} \mathbf{J} \cdot d \mathbf{s}+\int_{s} \frac{\partial \mathbf{D}}{\partial t} \cdot d \mathbf{s}+\sum_{i} \frac{d q_{i}}{d t}
\end{array}\right.
$$

For the moving electronic space, the relativistic effect will lead to the enhancement of electrical and magnetic effects.

Singularities also exist in the gravitational field. For example, there is a singularity in a black hole, which is a matter particle. It is divergent, because the curvature is also divergent. According to general relativity:

$$
\lim R_{\mu v ; \tau}^{\lambda}=\lim \left(\Gamma_{\mu \nu ; \tau}^{\lambda}+\Gamma_{\mu \tau ; v}^{\lambda}-\Gamma_{\alpha \beta}^{\lambda} \Gamma_{v \tau}^{\alpha}+\Gamma_{\alpha \tau}^{\lambda} \Gamma_{\beta v}^{\alpha}\right) \rightarrow \infty
$$

The energy momentum tensor is also divergent:

$$
\lim T_{\mu v} \rightarrow \infty
$$

Since the role of many trace elements in the gravitational field is equivalent to that of gravity, the singularity in the gravitational field is equivalent to the composition of many trace elements in the gravitation, and it is divergent.

\section{Conclusion}

In short, non-conservative phenomena are common in physics, and they obey the same law: energy is divergent, and it cannot obey time reversal invariance. 


\section{Acknowledgments}

I am very grateful to my physics teacher Zhang Xuefeng and my friend Ren Junyu for discussing with me.

\section{References}

[1] The department of mathematics in TongJi University. Higher Mathematics. Beijing: Higher Education Press; 2014.

[2] Yang Weihong. Mechanics and Theoretical Mechanics. Beijing: Science Press; 2014.

[3] Zhao Kaihua. Mechanics. Beijing: Higher Education Press; 2004.

[4] Pu Jin, Yang Shuzheng, Lin Kai. Lorentz-violating theory and tunneling radiation characteristics of Dirac particles in curved spacetime of Vaidya black hole. Acta Physics Sinica. 2019; 68(19): 21-26. 